\title{
Long term efficacy and safety of rivaroxaban plus cilostazol in the treatment of critical ischemia of the lower limbs in a frail, elderly patient with non valvular atrial fibrillation
}

\author{
Antonio Trani', Pietro Benedetto', Ferdinando Di Leo ${ }^{1}$, Angela Baiano ${ }^{1}$, Andrea Esposito ${ }^{1}$, Danilo Menna', \\ Arianna Allegretti ${ }^{2}$, Pierluigi Antonino Cappiello ${ }^{1}$ and Domenico Dell'Edera ${ }^{2^{*}}$ (D)
}

\begin{abstract}
Background: Many patients with critical lower limb ischemia are not eligible for revascularization procedures. Still, given the emerging role of both platelet and coagulation activation in the formation of arterial thrombi, they may benefit from the novel anticoagulant and antiplatelet drugs.

Case presentation: We describe the case of a male with critical lower limb ischemia complicated by older age, frailty, polymorbidity and non valvular atrial fibrillation, who was deemed as non eligible for surgery. The patient was successfully treated with the combination of rivaroxaban and cilostazol, and the clinical benefit was maintained throughout 32 months, with no occurrence of major or minor hemorrhagic or thrombotic events.

Conclusions: To our knowledge, this is the first report on the efficacy and safety of such a combination therapy in critical lower limb ischemia. In a clinical setting in which alternative pharmacological approaches are urgently needed, the association of rivaroxaban and cilostazol warrants further investigations.
\end{abstract}

Keywords: Cilostazol, Critical lower limb ischemia, Elderly, Non valvular atrial fibrillation, Rivaroxaban

\section{Background}

Currently, the treatment of critical lower limb ischemia, a sequelae of peripheral arterial disease (PAD), remains a challenge [1]. Despite the progress in revascularization procedures, many patients present multiple comorbidities, frailty (i.e., age $>80$ years, body weight $<60 \mathrm{~kg}$ and HAS-BLED $\geq 3$ ), and severe, diffuse lower limb atherosclerosis, which hamper their eligibility to surgical, endovascular or hybrid procedures [1, 2]. Yet, they may

\footnotetext{
* Correspondence: domenicodelledera68@gmail.com

${ }^{2}$ Cytogenetic and Molecular Genetics Unit, "Madonna delle Grazie" Hospital, 75100 Matera, Italy

Full list of author information is available at the end of the article
}

benefit from the novel anticoagulant and vasoactive antiplatelet drugs [3, 4], as both platelet and coagulation activation participate in the formation of arterial thrombi [4]. Thus, in patients with arterial diseases, the indication for antiplatelet therapy (recommended in first line) does not exclude anticoagulation [3-5]; rather, the association may be even superior to the antiaggregant agent alone, as shown by the recent COMPASS trial [5]. Indeed, this phase III study, which was prematurely stopped for overwhelming efficacy when only one half of the primary end-point events had occurred [6], showed the superiority of rivaroxaban plus aspirin, compared to aspirin alone, in reducing the risk of cardiovascular 
death, stroke, or myocardial infarction in patients with a history of stable atherosclerotic vascular disease. Rivaroxaban belongs to the class of the direct oral anticoagulants (DOACs), currently considered an advantageous alternative to the standard oral anticoagulants (i.e., the vitamin $\mathrm{K}$ antagonists, Vitamin $\mathrm{K}$ antagonists). Notably, DOACs, and, in particular, rivaroxaban, have yielded an acceptable benefit/risk ratio in patients with different venous and arterial thromboembolic diseases even when antiaggregants were required $[7,8]$.

Among antiplatelet agents, cilostazol, a potent phosphodiesterase type III selective inhibitor, is the only vasoactive and antiproliferative drug with documented evidence (grade IA) in the treatment of PAD when associated to traditional antiplatelet drugs [9]. Cilostazol has been shown to improve the walking distance in patients with intermittent claudication (Fontaine's stage IIA or IIB) [10], and is now recommended as the first choice in lower extremity PAD up to advanced stage IIB. Moreover, it consistently reduced restenosis/reocclusion after surgical and endovascular interventions in patients with critical ischemia, providing middle long term efficacy and decreased risk of hemorrhagic complications [11, 12]. In patients undergoing femoropopliteal stenting, cilostazol significantly reduced the rate of restenosis/ reocclusion and improved the primary patency rate, especially in subjects at high risk of in stent restenosis [13]. Moreover, it is the only agent that demonstrates, in randomized controlled trials, adequate efficacy in decreasing the occurrence of restenosis/reocclusion after coronary or peripheral revascularization procedures [14].

Is known that in elderly patients, the increase in the number of antiaggregant and anticoagulant drugs increases the rate of hemorrhagic events.

Some important studies document how this risk is reduced with the use of cilostazol provided that the therapy is personalized and the dosages in high risk cases are the minimum expected $(50 \mathrm{mg}$ twice daily for cilostazole and $15 \mathrm{mg}$ daily for rivaroxaban in patients with CAD and PAD and non-valvular atrial fibrillation) [15-20].

Moreover, cilostazole is not a traditional antiaggregant but is now considered by the scientific community as a drug "also antiaggregant" and the only one with strong evidence of anti-inflammatory, anti-restenosis and with current indications in the treatment of brain hemorrhage.

Given that antiaggregants are not contraindicated in patients already on DOACs because of non valvular atrial fibrillation (NVAF) or venous thromboembolic disease, and in those with carotid, coronary or peripheral stents, rivaroxaban and cilostazol might be good candidates for the treatment of patients with critical limb ischemia not eligible for surgery [21].

COMPASS study $[22,23]$ and VOYAGER-PAD study [24], stress the importance the association rivaroxaban with ASA in the prevention of ischemic limb events and in the substantial reduction of the rate of major amputations (about 70\%).

Here, we report the efficacy and safety of rivaroxaban plus cilostazol in a case of critical lower limb ischemia not suitable for revascularization, complicated by NVAF, frailty, older age and several comorbidities. Data for 32 months of follow-up are presented.

This association has shown that low dosages of both molecules $(15 \mathrm{mg} /$ day of rivaroxaban and $50 \mathrm{mg} / 2 /$ day of cilostazole), it resulted in limb salvage and prevented death from acute heart failure. The patient died at the age of 88 following viral pneumoni.

\section{Case report}

In January 2015, an 86-year-old male was admitted to our Hospital for critical ischemia of lower limbs characterized by rest pain, cyanosis on feet, acral ulcers, hypothermia below the knee socks and partial sensorymotor peripheral neuropathy. The patient, a former smoker, presented with frailty and multiple comorbidities, including multi-infarct dementia, previous recurrent transient ischemic attacks (TIA), chronic ischemic heart disease with three-vessel coronary artery disease deemed as non eligible for surgical intervention in 2013, NonValve Atrial Fibrillation (NVAF), dyslipidemia, chronic obstructive pulmonary disease (COPD), megaloblastic anemia, prior gastrectomy for gastric ulcer, and mild-tomoderate chronic kidney disease. The patient was not diabetic.

Drug therapy adopted was:

- dyslipidemia being treated with atorvastatin $(20 \mathrm{mg} / \mathrm{ay})$;

- arterial hypetension being treated with zofenopril (30 mg/day), lercanidipine hydrochloride (10 mg / day) and nitroglycerin in transdermal patches (5 mg/day).

- megaloblastic anemia being treated with a supplement based on folate (acid (6S) -5methyltetrahydrofolic), cyanocobalamin (vitamin B12), pyridoxine (vitamin B6), riboflavin (vitamin B2), betaine and zinc

- chronic obstructive pulmonary disease (BPCO) being treated with glycopyrronium bromide (one inhalation/day).

The patient was unsuitablenot suitable for myocardial (CAD) and peripheral (PAD) revascularization, given the remarkable severity and diffusion of atherosclerotic pathology.

Cholesterol and triglyceride parameters before using atorvastatin were: total cholesterol $250 \mathrm{mg} / \mathrm{dl}$, HDL cholesterol $45 \mathrm{mg} / \mathrm{dl}$, LDL cholesterol $181 \mathrm{mg} / \mathrm{dl}$, triglycerides $120 \mathrm{mg} / \mathrm{dl}$. After use of atorvastatin the values have 
significantly decreased: total cholesterol $137 \mathrm{mg} / \mathrm{dl}$, HDL cholesterol $57 \mathrm{mg} / \mathrm{dl}$, LDL cholesterol $70 \mathrm{mg} / \mathrm{dl}$ and triglycerides $51 \mathrm{mg} / \mathrm{dl}$.

The patient underwent echo color-Doppler and continuous-wave Doppler examination of lower limbs, which revealed multiple steno-obstructive lesions of the iliac, femoropopliteal and tibial segments, and anklebrachial index (ABI) of approximately $0.3-0.35$, bilaterally. Computed tomography $(\mathrm{CT})$ angiography of the aorta, iliac arteries, and lower limb arteries showed extensive fibrocalcific atherosclerotic plaques in the iliac, femoropopliteal and tibial segments, bilaterally, deemed as non-eligible for surgical, endovascular or hybrid procedures. Moreover, CT-angiography of supra aortic vessels and cerebral circulation displayed the presence of multiple severe steno-obstructive lesions of both extraand intra-cranial arteries.

Treatment with intravenous heparin and antiplatelet drugs (acetylsalicylic acid $100 \mathrm{mg} /$ day and clopidogrel $75 \mathrm{mg}$ /day) was commenced.

After 10 days, the patient was discharged with the indication for anticoagulant and antiplatelet therapy with warfarin (international normalized ratio [INR]: 2-3) and clopidogrel $75 \mathrm{mg} /$ day. As, over the following 3 months, the INR ranged between 2 and 3.5 in only $45 \%$ of evaluations, the patient was switched to rivaroxaban $15 \mathrm{mg} /$ day plus cilostazol, which was started at the dose of $150 \mathrm{mg} /$ day and then increased up to $200 \mathrm{mg} /$ day. The choice of the low dose of rivaroxaban (vs. $20 \mathrm{mg} /$ day) was made based on the advanced age, frailty, body weight $(<60 \mathrm{~kg})$ and creatinine clearance (between 15 and $49 \mathrm{ml} / \mathrm{min}$ ) of the patient $[25,26]$.

At the following visits, $6,12,18,24$ and 30 months after discharge, the clinical and echo color doppler examinations confirmed improved revascularization, with acceptable perfusion of lower limbs, complete healing of acral ulcers present at both admission and discharge, and a clear amelioration of ischemia, disappearance of rest pain (from Fontaine's stage III to non-advanced stage IIB, with $>100 \mathrm{~m}$ of functional autonomy) and improvement of the ABI (from approximately 0.3 [left] and 0.35 [right] before the start of rivaroxaban and cilostazol, to 0.55 [left] and 0.6 [right] afterwards). Such results were maintained over time, in the absence of progression of the other cardiovascular and metabolic diseases and pre-existing conditions.

Approximately 32 months after the start of rivaroxaban plus cilostazol, although several assessments and dose adjustments were required with regard to the medications for concomitant diseases, limb perfusion was still good (the limb was saved), the hematochemical parameters (blood count, coagulation, serum creatinine, and liver function) were stable and no major or minor hemorrhagic or thrombotic events had occurred.
Unfortunately, soon after the last follow-up visit, the patient, who suffered from advanced COPD, died of acute respiratory insufficiency due to severe pulmonary infection. The cause was, in no way, related to the treatment for vascular disease and, indeed, no signs of bleeding were documented.

Furthermore, the patient presents a CHADS2 score (risk of cerebral infarction in patients with atrial fibrillation) equal to 5 and HAS-BLED score (risk of bleeding) equal to 4 .

\section{Discussion}

To date, the presence of diffuse steno-obstructive lesions in the arterial occlusive disease of lower limbs, and, in particular, critical lower limb ischemia, makes affected patients frequently non-eligible for traditional surgery procedures or novel molecular and cellular therapies (i.e., growth factors, stem cells, and stem cells from adipose tissue) $[27,28]$. This is especially true in the case of frail patients aged $>80$ years, who often suffer from multiple diseases. Therefore, alternative pharmacological approaches are urgently needed for the management of these subjects [29].

Here, we report the successful treatment with rivaroxaban plus cilostazol of a patient with critical lower limb ischemia complicated by older age, frailty, and several concomitant cardiovascular and metabolic diseases, including NVAF. Notably, after 32 months, the patient was still in good clinical conditions, without major or minor hemorrhagic or thrombotic events. To the best of our knowledge, this is the first demonstration of the efficacy and safety of such a combination therapy in critical lower limb ischemia. Our results are even more encouraging considering the long follow-up period and the complex clinical conditions of the patient, which are frequently encountered by physicians in their daily practice. Notably, Ozker and coworkers reported on the usefulness of rivaroxaban and cilostazol in a dysfibrinogenemic patient with thrombotic episodes, providing additional evidence that this combination may represent a therapeutic option in certain cases, that warrants further investigations.

As arterial thromboembolic events rely on both pro aggregant and pro thrombotic mechanisms, patients should always be administered anticoagulant and antiplatelet vasoactive agents. In this way, the different steps of primary arterial thrombosis and reocclusion/restenosis can be successfully inhibited. In daily practice, the skilled vascular surgeon/angiologist faces the choice of the most adequate therapeutic strategy based on novel combinations of anticoagulant and vasoactive antiplatelet drugs (possibly feasible also at home). The treatment must maximize the effect on the collateral arterial, muscular and skin circulation, and make these vessel 
networks more complex and effective to guarantee the most active alternative microcirculation in order to save the limb and prolong patient survival. In this context, a major role may be played by the well documented vasoactive and antiproliferative features of cilostazol, when combined with the antiplatelet and thrombolytic properties of rivaroxaban and its effects on arterial circulation. Such approach allowed to treat the thrombosis of small medium sized arterial vessels (representing the core of collateral circulations) in our case as in previous reports. This hypothesis is corroborated by the complex case of critical lower limb ischemia described here, in which the association of cilostazol and rivaroxaban has positively affected the outcome of artery disease.

At present, the results of the RCT VOYAGER PAD study (ClinicalTrials.gov Identifier: NCT02504216) are eagerly awaited, as it is the only randomized controlled trial (RCT) exploring the efficacy and safety of rivaroxaban in symptomatic PAD following peripheral revascularization procedures of the lower extremities. However, several groups, including ours, have acknowledged the pivotal role of rivaroxaban (sometimes used off-label) as adjuvant therapy after surgical or endovascular procedures in maintaining the efficacy of the intervention and in the salvage of the limb. In our experience, adding cilostazol to rivaroxaban has positively impacted on patient outcome, even after 32 months.

\section{Conclusion}

Success in treating critical limb ischemia is measured by amputation-free survival (AFS). Although patients with critical limb ischemia are revascularized by endovascular or surgical procedures, amputation rates remain high. One year after the onset of critical limb ischemia approximately $25 \%$ of patients will have to undergo major limb amputation. While about $35-67 \%$ will experience major limb amputation within 4 years. Additionally, early post-operative mortality rates vary from 4 to $22 \%$ after major limb amputation [30]. Numerous studies suggest that a polypharmacological approach is needed.

- anticoagulant or antithrombotic associated with anti Xa drugs (rivaroxaban) (fibrillating or nonfibrillating patients).

- antiplatelet agent (acetylsalicylic acid or clopidogrel) in associated coronary artery disease.

- Vasoactive (cilostazol), which plays an important role in the inhibition of proilferative cell replication processes also involved in the evolution of atherosclerosis and in the pathogenesis of restenosis.

These drugs certainly increase bleeding risk but not significantly if used at appropriate dosages. It is clear that this approach must always take into account the benefit/risk ratio and the degree of reversibilityirreversibility of the ischemic framework.

The case report described here supports the association of rivaroxaban and cilostazol as a useful strategy to treat critical lower limb ischemia in patients non-eligible for revascularization procedures and encourages further investigations. In the absence of compelling evidence from RCTs, case reports, which represent the first line of evidence, are crucial to collect data on the efficacy and safety of novel therapeutic strategies.

\section{Supplementary information}

Supplementary information accompanies this paper at https://doi.org/10. 1186/s40780-020-00173-9.

\section{Additional file 1.}

\section{Abbreviations}

PAD: Peripheral arterial disease; HAS-BLED: Hypertension abnormal renal and liver function stroke bleeding labile inr elderly drugs; DOACs: Direct oral anticoagulants; VKAs: Vitamin K antagonists; NVAF: Valvular atrial fibrillation; COPD: Chronic obstructive pulmonary disease; ABI: Ankle-brachial index; $C T$ : Computed tomography; INR: International normalized ratio; $\mathrm{RCT}$ : Randomized controlled trial;

\section{Acknowledgments}

The authors would like to thank Clara Ricci, Ph.D., Raquel Carvalhosa, Ph.D., and ERA ms for editorial assistance.

\section{Authors' contributions}

AT wrote the manuscript. AT and DD conceived and designed the study. PB, $A A$ and $P B$ were responsible for the collection and analysis of the experimental data. FD and AE interpreted the data and drafted the manuscript. $A B$ and $P A C$ revised the manuscript critically for important intellectual content. All authors read and approved the final manuscript. Ethics approval and consent to participate The study was approved by the Ethics Committee of Qingpu Branch of Zhongshan Hospital Affiliated to Fudan University (Shanghai, China). Patients who participated in this research had complete clinical data. Signed informed consents were obtained from the patients or the guardians.

\section{Funding}

Not applicable.

\section{Availability of data and materials}

All data generated or analyzed during this study are included in this published article.

Ethics approval and consent to participate

Not applicable.

\section{Consent for publication}

Written informed consent was obtained from the patient for publication of this case report -Chief of this journal.

\section{Competing interests}

The authors declare that they have no competing interests.

\section{Author details}

'Vascular and Endovascular Surgery Unit, Cardiovascular Department, San Carlo Hospital Potenza, via Potito Petrone, 85100 Potenza, Italy. ${ }^{2}$ Cytogenetic and Molecular Genetics Unit, "Madonna delle Grazie" Hospital, 75100 Matera, Italy. 
Received: 3 April 2020 Accepted: 18 June 2020

Published online: 03 August 2020

\section{References}

1. Farber A, Eberhardt RT. The current state of critical limb ischemia: a systematic review. JAMA Surg 2016; 151: 1070

2. Martini $R$, Andreozzi GM, Deri A, et al. Amputation rate and mortality in elderly patients with critical limb ischemia not suitable for revascularization. Aging Clin Exp Res. 2012;24:24-7.

3. Stephan D, Cordeanu E-M, Mirea C, et al. Place of non-vitamin K antagonist oral anticoagulants in anticoagulant-antiplatelet combinations in peripheral artery disease. Arch Cardiovasc Dis. 2016;109:634-40.

4. Hess CN, Norgren L, Ansel GM, et al. A structured review of antithrombotic therapy in peripheral artery disease with a focus on revascularization: a TASC (InterSociety consensus for the Management of Peripheral Artery Disease) initiative. Circulation. 2017;135:2534-55.

5. Eikelboom JW, Connolly SJ, Bosch J, et al. Rivaroxaban with or without aspirin in stable cardiovascular disease. N Engl J Med. 2017;377:1319-30.

6. Braunwald E. An important step for Thrombocardiology. N Engl J Med. 2017:377:1387-8.

7. Heidbuchel $H$, Verhamme $P$, Alings $M$, et al. Updated European heart rhythm association practical guide on the use of non-vitamin $\mathrm{K}$ antagonist anticoagulants in patients with non valvular atrial fibrillation. Europace. 2015;17:1467-507.

8. Kubitza D, Berkowitz SD, Misselwitz F. Evidence-based development and rationale for once-daily rivaroxaban dosing regimens across multiple indications. Clin Appl Thromb. 2016;22:412-22.

9. Gerhard-Herman MD, Gornik HL, Barrett C, et al. 2016 AHA/ACC guideline on the Management of Patients with Lower Extremity Peripheral Artery Disease: executive summary: a report of the American College of Cardiology/American Heart Association task force on clinical practice guidelines. Circulation; 135 Epub ahead of print 21 March 2017. DOI: https:// doi.org/10.1161/CIR.0000000000000470.

10. Bedenis R, Stewart M, Cleanthis M, et al. Cilostazol for intermittent claudication. Cochrane Database Syst Rev. Epub ahead of print 31 October 2014. DOl: https://doi.org/10.1002/14651858.CD003748.pub4.

11. Soga $Y$, lida $O$, Hirano $K$, et al. Impact of cilostazol after endovascular treatment for infrainguinal disease in patients with critical limb ischemia. J Vasc Surg. 2011:54:1659-67.

12. Rigatelli G, Shah SR, Arshad A, et al. Medical Therapy for Critical Limb Ischemia. In: Dieter RS, Dieter Jr RA, Dieter III RA, et al., editors. Critical Limb Ischemia. Cham: Springer International Publishing. p. 537-42.

13. Iftikhar $\mathrm{O}$, Oliveros $\mathrm{K}$, Tafur AJ, et al. Prevention of Femoropopliteal in-stent restenosis with Cilostazol: a meta-analysis. Angiology. 2016;67:549-55.

14. de Donato $\mathrm{G}$, Setacci $\mathrm{F}$, Mele $\mathrm{M}$, et al. Restenosis after coronary and peripheral intervention: efficacy and clinical impact of Cilostazol. Ann Vasc Surg. 2017:41:300-7

15. Ozker E, Atalay F, Gulmez O, Saritas B. Treating a patient of Dysfibrinogenemia with acute thromboembolism by rivaroxaban and Cilostazol. Indian J Hematol Blood Transfus. 2017:33(3):431-3.

16. Spinthakis N, Farag M, Rocca B, Gorog DA. More, more, more: reducing thrombosis in acute coronary syndromes beyond dual antiplatelet therapy_current data and future directions. J Am Heart Assoc. 2018;7(3): e007754.

17. Zavgorodnyaya D, Knight TB, Daley MJ, Teixeira PG. Antithrombotic therapy for postinterventional management of peripheral arterial disease. Am J Health Syst Pharm. 2020;77(4):269-76.

18. Real J, Serna MC, Giner-Soriano M, Forés R, Pera G, Ribes E, Alzamora M, Marsal JR, Heras A, Morros R. Safety of cilostazol in peripheral artery disease: a cohort from a primary healthcare electronic database. BMC Cardiovasc Disord. 2018;18(1):85

19. Disdier Moulder MPA, Hendricks AK, Ou NN. Towards appropriate polypharmacy in older cardiovascular patients: how many medications do I have to take? Clin Cardiol. 2020;43(2):137-44.

20. Daimon S. Adverse effect of antithrombotic medications on bleeding events and comparison of antithrombotic agents in hemodialysis patients. Ther Apher Dial. 2019;23(1):32-37. 14.

21. Björck M, Earnshaw JJ, Acosta S, Gonçalves FB, Frederic C, Debus ES, Hinchliffe R, Jongkind V, Koelemay MJW, Menyhei G, Svetlikov AV, Tshomba Y, Van Den Berg JC. Editor's choice-European Society for Vascular Surgery
(ESVS) 2020 clinical practice guidelines on the Management of Acute Limb Ischaemia. Eur J Vasc Endovasc Surg. 2020:59(2):173-218.

22. Gurbel PA, Fox KAA, Tantry US, Cate HT, Weitz Jl. Combination antiplatelet and oral anticoagulant therapy in patients with coronary and peripheral artery disease focus on the COMPASS Trial. Circulation. 2019;139:2170-85.

23. Hussaina MA, Wheatcrofta M, Nault P, Lindsay TF, Bhatt DL, Anand SS, Verma S, Al-Omran M. COMPASS for vascular surgeons: practical considerations. Curr Opin Cardiol. 2019;34(2):178-84.

24. Debus ES, Nehler MR; Executive committee of the Voyager PAD trial. The voyager PAD trial - new path for post-revascularisation PAD patients. Eur J Vasc Endovasc Surg. 2020. pii: S1078-5884(20)30276-8.

25. Kirchhof P, Benussi S, Kotecha D, et al. 2016 ESC guidelines for the management of atrial fibrillation developed in collaboration with EACTS. Eur J Cardiothorac Surg. 2016;50:e1-e88.

26. Ozker E, Atalay F, Gulmez O, et al. Treating a patient of Dysfibrinogenemia with acute thromboembolism by rivaroxaban and Cilostazol. Indian J Hematol Blood Transfus. 2017;33:431-3.

27. Perzborn E, Heitmeier S, Laux V. Effects of rivaroxaban on platelet activation and platelet-coagulation pathway interaction: in vitro and in vivo studies. J Cardiovasc Pharmacol Ther. 2015:20:554-62.

28. Bánovčin P, Škorňová I, Samoš M, et al. Platelet aggregation in direct Oral factor Xa inhibitors-treated patients with atrial fibrillation: a pilot study. J Cardiovasc Pharmacol. 2017;70:263-6

29. Lee $\mathrm{H}-\mathrm{F}$, Chan $\mathrm{Y}-\mathrm{H}$, Wang $\mathrm{C}-\mathrm{L}$. Using rivaroxaban as thrombolytic treatment for a patient of pedal arch arterial thrombosis with suboptimal result of endovascular therapy. Acta Cardiol Sin. 2016:32:623-6.

30. Duff $S$, Mafilios MS, Bhounsule $P$, Hasegawa $T$. The burden of critical limb ischemia: a review of recent literature. Vasc Health Risk Manag. 2019;15:187-208.

\section{Publisher's Note}

Springer Nature remains neutral with regard to jurisdictional claims in published maps and institutional affiliations.

Ready to submit your research? Choose BMC and benefit from:

- fast, convenient online submission

- thorough peer review by experienced researchers in your field

- rapid publication on acceptance

- support for research data, including large and complex data types

- gold Open Access which fosters wider collaboration and increased citations

- maximum visibility for your research: over $100 \mathrm{M}$ website views per year

At $\mathrm{BMC}$, research is always in progress.

Learn more biomedcentral.com/submissions 